Check for updates

Cite this: RSC Adv., 2019, 9, 5431

\title{
Recycling of vitrimer blends with tunable thermomechanical properties $\uparrow$
}

\author{
Biao Zhang,,$^{\text {ab }}$ Chao Yuan, $\ddagger^{a}$ Wang Zhang, ${ }^{\text {ac }}$ Martin L. Dunn, ${ }^{\text {ad }} H$. Jerry Qi, (D) e \\ Zhuangjian Liu, ${ }^{f}$ Kai Yu (DD *d and Qi Ge (DD*ac
}

Vitrimers are a new class of thermosetting polymers that can be thermally processed through bond exchange reactions (BERs) without losing network integrity. In engineering applications, the tunability of their thermomechanical properties is highly desirable to meet the requirements of different working conditions. Here, we report a simple composite-based strategy that avoids complex chemistry to prepare vitrimer blends with tunable thermomechanical properties by virtue of the good weldability of base vitrimers. Effects of processing parameters (such as temperature and time) on the properties of recycled vitrimer blends are experimentally investigated. A computational model that accounts for the random distribution of component vitrimer particles is developed to predict the thermomechanical properties of the recycled vitrimer blends with various compositions. Good agreement is achieved between theoretical prediction and experiment. Parametric studies are further conducted by employing the computational model to explore the designability and provide some basic principles to guide the design of recycled vitrimer blends. Reasonable recyclability of the vitrimer blends is verified by multiple generations of recycling experiments.

Received 2nd January 2019
Accepted 31st January 2019

DOI: $10.1039 /$ c9ra00015a

rsc.li/rsc-advances
Recently, a new class of thermosetting polymers, known as vitrimers, which can be thermally processed without losing network integrity through bond exchange reactions (BERs), has changed the traditional view of thermosetting polymers. ${ }^{11,12}$ Vitrimers behave like traditional thermosets at room temperature, while tend to be reshapable, reprocessable, and recyclable at elevated temperatures due to the inherent dynamic covalent bonds (DCBs). ${ }^{12-17}$ These innovative features possessed by vitrimers expand the lifetime and potential applications of thermosetting polymers. ${ }^{13}$ Since Leibler and coworkers first introduced the name vitrimers for the epoxy networks which were developed based on transesterification reaction in 2011, several alternative chemistries have been explored. ${ }^{18-29}$

The tunability of the thermomechanical properties of the vitrimers is highly desirable in engineering applications to meet the requirements of different working conditions. For instance, when an epoxy-based vitrimer is used as a structural material, we normally expect to increase its modulus at room temperature to meet the requirement of load-bearing capacity; on the other hand, decreasing its rubbery modulus eases the reprocessing at high temperature. However, for the existing chemistrybased methods, such as adjusting the stoichiometry of monomers ${ }^{30}$ or choosing different monomers, ${ }^{31,32}$ increasing roomtemperature modulus and decreasing rubbery modulus of vitrimers are mutually exclusive since the cross-linking density or the rigidity of the entire network is tuned. Therefore, it is necessary to further explore other methods to expand the tunability of the thermomechanical properties of vitrimers.

$\dagger$ Electronic supplementary information (ESI) available. See DOI: 10.1039/c9ra00015a

‡ These authors contributed equally to this work. 
Furthermore, since vitrimers possess the virtue of recyclability, from both environmental and economic points of view, it is valuable that new vitrimers with different mechanical performances can be fabricated by reprocessing the "waste" vitrimers, rather than involving complex chemistry.

In this work, we report a composite-based strategy to prepare vitrimer blends with tunable thermomechanical properties utilizing the good weldability of vitrimers. Two different transesterification-based epoxy vitrimers were respectively synthesized by reactions of diglycidyl ether of bisphenol A (DGEBA) with fatty acids and glutaric anhydride, showing different thermomechanical properties. ${ }^{11}$ The resulting vitrimers were pulverized into powders followed by being mixed at different ratios to prepare vitrimer blends under reprocessing conditions. The transesterification-based property of both base vitamers facilitates the interfacial welding of mixed vitrimer particles. Dynamic mechanical analysis (DMA) and uniaxial tensile test were carried out to investigate the effect of processing parameters (such as temperature and treating time) on the thermomechanical properties of recycled vitrimer blends. By simply varying the composition of the two base vitrimers in their pulverous states, the thermomechanical properties of the recycled vitrimer blends were tuned within the limits regulated by the pure base vitrimers. To better understand the operative phenomena and assist the design of vitrimer blends, a computational model accounting for the random distribution of component vitrimer particles was developed. Parametric studies were further conducted by employing the model to explore the designability of the vitrimer blends. In addition, recyclability of the vitrimer blends was investigated through multiple generations of recycling experiments.

\section{Experimental}

\subsection{Material synthesis}

Two epoxy-based vitrimers were synthesized according to the reported procedures (Fig. S1 $\dagger$ ), ${ }^{\mathbf{1 1}, \mathbf{1 2}}$ where monomers with different chain lengths were used to generate their distinction in crosslinking density. Accordingly, these two vitrimers have different glass transition temperatures $\left(T_{\mathrm{g}}\right)$ and roomtemperature modulus, which were regarded as soft and hard networks. But both of them ran transesterification at elevated temperature. Details regarding the synthetization can be found in the ESI. $\dagger$

\subsection{Preparation of powders}

Bulk samples of the two aforementioned pure vitrimers were respectively grinded into powders by using a variable speed rotor mill branded PULVERISETTE 14 (Fritsch, Germany) equipped with a trapezoidal perforation sieve with ring size of $0.5 \mathrm{~mm}$. The grinded particles were then transferred to a digital microscope (Hirox, Japan) for morphology observation.

\subsection{Preparation of recycled samples}

Vitrimer powders were prepared by first mixing the two types of powders in different weight ratios followed by pouring the powder mix into a customized aluminum mold. Then we preheated the CARVER press (Model 4386, USA) machine to the target temperature and transferred the mold into the machine chamber where the mixed powders were hot-pressed for a set time under constant pressure.

\subsection{Dynamic mechanical analysis}

Dynamic mechanical analysis (DMA) tester (Q800, TA Instruments, New Castle, DE, USA) was used to characterize the thermomechanical properties of the vitrimer blend samples in the tension mode. Samples with the dimension of $15 \mathrm{~mm} \times$ $5 \mathrm{~mm} \times 0.5 \mathrm{~mm}$ were tested at a frequency of $1 \mathrm{~Hz}$ with a peakto-peak amplitude of $2 \%$. The temperature was first equilibrated at $-50{ }^{\circ} \mathrm{C}$ for $5 \mathrm{~min}$, and then gradually increased to $120^{\circ} \mathrm{C}$ at a rate of $2{ }^{\circ} \mathrm{C} \min ^{-1}$. The glass transition temperature $\left(T_{\mathrm{g}}\right)$ was identified as the temperature corresponding to the peak of loss factor $(\tan \delta$ ) curve.

\subsection{Uniaxial tension tests}

For the room-temperature uniaxial tension tests, the universal material test machine Instron (Model 5943, Germany) was used in the displacement-controlled mode with a load cell of $1 \mathrm{kN}$. Samples with the dimension of $15 \mathrm{~mm} \times 5 \mathrm{~mm} \times 0.5 \mathrm{~mm}$ were tested at a loading rate of $5 \% \mathrm{~min}^{-1}$ to minimize viscoelastic effects. For each case, three samples were tested, and the average value was reported. For the tests at high temperature $\left(100{ }^{\circ} \mathrm{C}\right)$, the DMA tester was used where the loading rate and sample dimension were the same as the tests performed at room temperature.

\section{Computational modeling}

To predict the thermomechanical properties of the recycled vitrimer blends with various compositions, a computational model was developed where the temperature-dependent thermomechanical behaviors of the pure vitrimers and the random distribution of the component vitrimer particles within the blends were both taken into account.

\subsection{Thermoviscoelastic model for the pure vitrimer}

For the pure vitrimers with hard or soft network, a multibranch thermoviscoelastic model was employed where one equilibrium branch and several thermoviscoelastic nonequilibrium branches were arranged in parallel (Fig. 1a). ${ }^{33,34}$ More details can be seen in the ESI. $\dagger$

\subsection{Finite element computational model}

Finite element (FE) simulations of the DMA test for the pure vitrimers were conducted to validate the accuracy of the above characterized material parameters by using the commercial software package ABAQUS (Dassault Systems, Johnston, RI, USA). A two-dimensional square representative volume element (RVE) constrained by periodic boundary conditions was built and assigned with the properties of pure vitrimer. The aforementioned multibranch thermoviscoelastic constitutive 


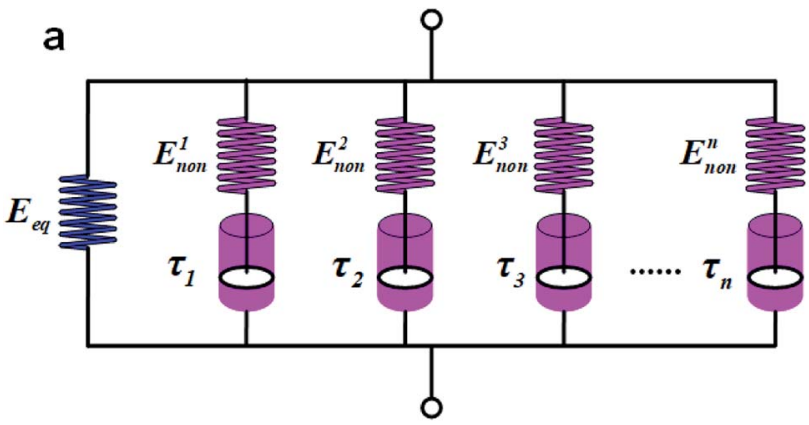

b

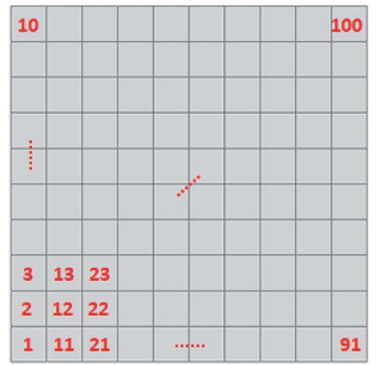

C

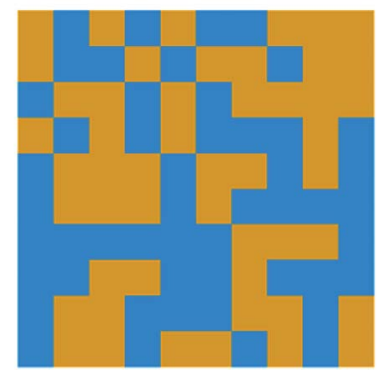

Fig. 1 (a) Schematic diagram of the multibranch thermoviscoelastic model. (b) Division of the representative volume element (RVE) where 100 square subzones are labelled from 1 to 100. (c) Representative volume element with material assignment where orange and blue sections respectively represent hard and soft vitrimers.

relations was programmed in the FE simulations by importing the fitted viscoelastic parameters (given in Table S1†) into the time-domain Prony-series provided in ABAQUS material library together with a UTRS subroutine to define the time-temperature superposition (TTSP) relationship. Four-node bilinear plane stress elements (ABAQUS element type CPS4) were employed to mesh the entire part. Dynamic, implicit analyses were conducted at elevated temperatures under a uniaxial sinusoidal oscillation strain at a frequency of $1 \mathrm{~Hz}$ with a peak-to-peak amplitude of $2 \%$. The calculated phase lag between the stress and strain curves determines the loss factor $(\tan \delta)$ while the storage modulus is taken to be the quotient of the stress and strain amplitudes. It is seen from Fig. S2 (FE simulation part in ESI $\dagger$ ) that good agreement is achieved between the experiment and simulation of the storage modulus and $\tan \delta$ for both hard and soft vitrimers, which validates the accuracy of the computation model.

For the simulations of the fully recycled polymer blends, we assume that the neighboring particles are well bonded and thus the effect of the interfacial imperfections can be neglected. Moreover, as analyzed in Fig. 2, no apparent difference was found in the average particle size between the soft and hard vitrimers (details can be seen in Results and discussion part). In this case, we assume the soft and hard powders possess the same particle size. With these assumptions and in order to balance computational complexity with microstructure model fidelity, we model the vitrimer blend microstructure by uniformly dividing the representative volume element (RVE) into 100 square subzones labelled from 1 to 100 (Fig. 1b). Each of the subzones is assigned with the material property of either
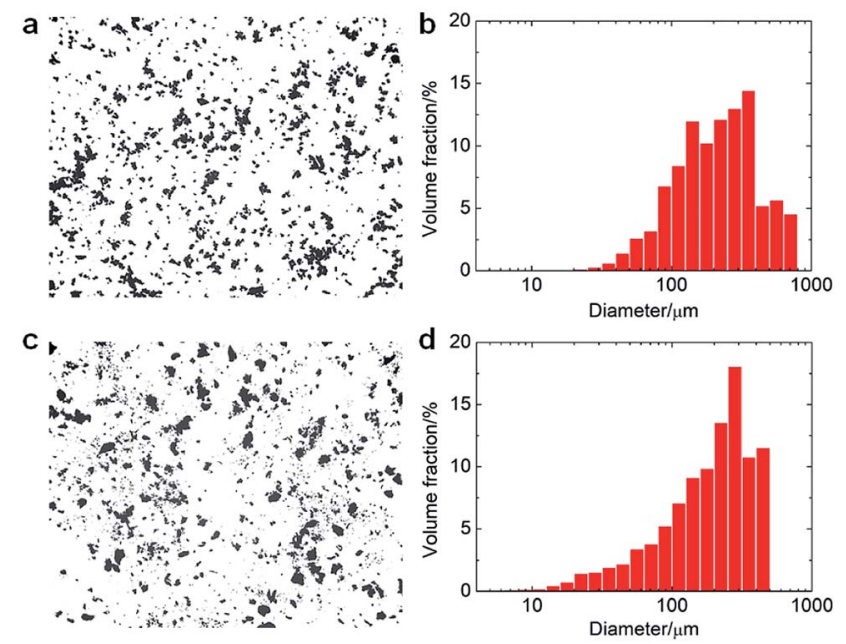

Fig. 2 (a) Microscope image of particles from soft networks and (b) the corresponding particle size distribution; (c) microscope image of particles from hard networks and (d) the corresponding particle size distribution.

the hard or soft vitrimer. Assume the composition of the hard epoxy is $n \%$ ( $n$ is an integer), a Matlab code was called to generate $n$ unduplicated random integers ranging from 1 to 100 , and the subzones labelled by these random numbers were assigned with the material properties of hard vitrimer. The rest subzones correspond to the soft vitrimer (Fig. $1 \mathrm{~b}$ and c). Similar to the simulation procedures mentioned for the pure vitrimer, the temperature dependent storage modulus and $\tan \delta$ of the vitrimer blends with various composition $(7: 3,5: 5$ and $3: 7)$ were predicted and the comparison between the experiment and simulation are shown in Fig. $4 \mathrm{a}$ and b.

\section{Results and discussion}

\subsection{Characterization of the polymer particles}

As the microscopic images shown in Fig. 2a and c, the particles grinded from soft and hard networks are both in irregular shapes with different sizes.

To further obtain the particle size distributions of the grinded powders from different vitrimers, microscope images were analyzed by using the commercial image processing software Image J. The profiles of the particles were extracted from the microscopic image and the size of each particle was taken to be the diameter of a circle with an equal area of the corresponding particle profile. Fig. $2 \mathrm{~b}$ and d show the particle size distributions of the grinded powders from soft network and hard network. The volume fraction of the particles with diameter ranging from $100 \mu \mathrm{m}$ to $400 \mu \mathrm{m}$ is calculated to be $70 \%$ for soft network and $68 \%$ for hard network, which indicates that with the same mesh size of sieve ring, there is no apparent difference in the average particle size between the soft and hard networks.

\subsection{Effects of treating time and temperature}

Since the transesterification reaction is time and temperature dependent, ${ }^{12}$ the effects of these two treating parameters on the 
thermomechanical performances of vitrimer blends were investigated.

To explore the effect of treating time, the mixed vitrimer powders (soft : hard = 1:1) were thermally treated at $200{ }^{\circ} \mathrm{C}$ under a controlled pressure of $16 \mathrm{MPa}$ for $20 \mathrm{~min}, 40 \mathrm{~min}, 1 \mathrm{~h}$, $2 \mathrm{~h}, 4 \mathrm{~h}$ and $6 \mathrm{~h}$, respectively. It is seen from Fig. $3 \mathrm{a}-\mathrm{c}$ that the increase of heating time from $20 \mathrm{~min}$ to $2 \mathrm{~h}$ results in a gradual increase of rubbery modulus from $\sim 4.7 \mathrm{MPa}$ to $\sim 10 \mathrm{MPa}$, which suggests that the voids in the vitrimer blends are gradually closed and more polymer chains are connected between particle interfaces. ${ }^{15,35}$ Above the heating time of $2 \mathrm{~h}$, the rubbery modulus becomes heating time-independent, indicating the interfacial chain density reaches a saturated level. In addition, two different glass transition temperatures of $\sim 28{ }^{\circ} \mathrm{C}$ and $\sim 75{ }^{\circ} \mathrm{C}$ could be found for the vitrimer blends and the values are barely affected by the heating time. Moreover, the uniaxial tension curves of the vitrimer blends at $100{ }^{\circ} \mathrm{C}$ (Fig. 3d) shows that an improved stretchability could be obtained by the vitrimer blends with an increasing heating time from $20 \mathrm{~min}$ to $4 \mathrm{~h}$ where the break stain increases from $6.5 \%$ to $15 \%$.

Previous work by $\mathrm{Yu}$ et $a .^{35}$ showed that the interfacial welding of vitrimer follows the Arrhenius-type TTSP. Therefore, increasing the heating temperature shows the same effect to improve the mechanical performance of the vitrimer blends as increasing the heating time. Detailed experimental results are shown in Fig. S3 of the ESI. $\dagger$

Overall, time and temperature will affect the extent of bond exchange reactions at the interfaces of vitrimer particles. With the increase of time or temperature, the interfacial chain density will increase, which therefore leads to the increase of modulus. However, when the time or temperature increases to a certain extent, the interfacial chain density becomes saturated, and correspondingly the modulus maintains constant.
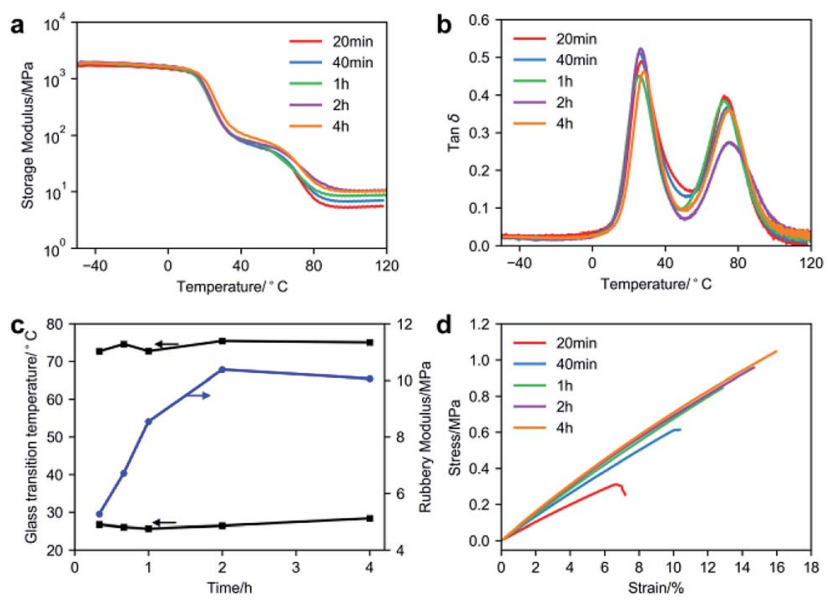

Fig. 3 Effect of heating time on thermomechanical performance of recycled vitrimer blends. Storage modulus (a) and $\tan \delta$ (b) as a function of temperature. (c) The effect of heating time on glass transition temperature and rubbery modulus. (d) Strain-stress behavior of recycled materials with $50 \%$ of hard network at $100{ }^{\circ} \mathrm{C}$ (rubbery state). All the samples were prepared by mixing $50 \%$ of soft network and $50 \%$ hard network polymer powders resulting from the utilization of $0.5 \mathrm{~mm}$ trapezoidal perforation sieve rings under the same controlled pressure of $16 \mathrm{MPa}$ at $200^{\circ} \mathrm{C}$.

\subsection{Effect of composition}

The vitrimer blends can be regarded as a composite whose mechanical properties approximately follows the rule of mixtures. Therefore, their thermomechanical properties could be tuned by simply varying the mixing ratio between the two base vitrimers. The temperature dependent storage modulus and $\tan \delta$ of the vitrimer blends with varying composition are respectively shown in Fig. $4 \mathrm{a}$ and $\mathrm{b}$, and the corresponding rubbery modulus and glass transition temperatures $\left(T_{\mathrm{g}} \mathrm{s}\right)$ are summarized in Fig. 4c. Simulation results obtained by the computational model are observed to agree well with the experiment results, which validates the accuracy of the developed model. It is seen from Fig. $4 \mathrm{c}$ that the rubbery modulus gradually increases from $1.1 \mathrm{MPa}$ to $18 \mathrm{MPa}$ with the weight ratio of hard vitrimer varying from $0 \%$ to $100 \%$.

In addition to the DMA results, uniaxial tension results shown in Fig. 4d and e further show the tunability of the stiffness and stretchability by varying the blend composition. It is seen in Fig. 4e that as the ratio of hard vitrimer increases, the Young's modulus of the vitrimer blends gradually increases from $3 \mathrm{MPa}$ to $1.15 \mathrm{GPa}$, along with the decrease of break strain from $200 \%$ to $10 \%$.
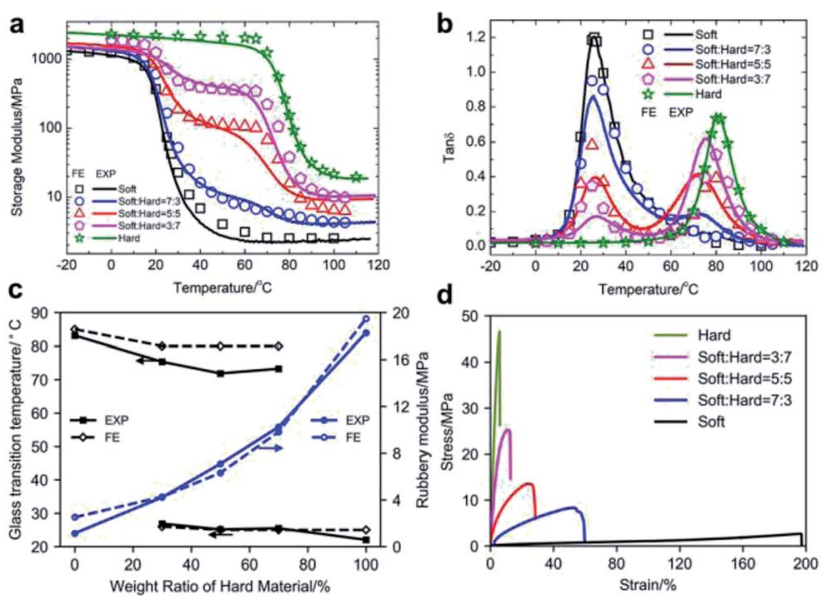

d
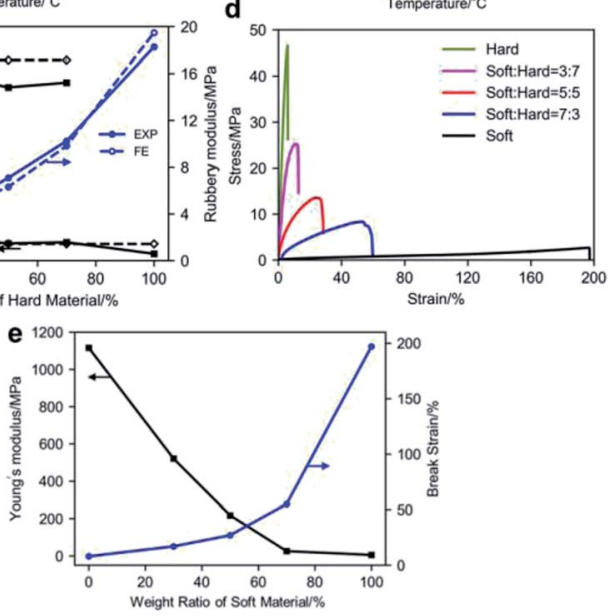

Fig. 4 Effect of composition on the thermomechanical performance of recycled vitrimer blends. Storage modulus (a) and $\tan \delta$ (b) as a function of temperature. Solid lines illustrate experimental results and dash lines denote the prediction by using the computational model. (c) The effect of composition on glass transition temperature and rubbery modulus. (d) Strain-stress behavior of vitrimer blends at room temperature. S: soft; $\mathrm{H}$ : hard. (e) The effect of composition on Young's modulus and break strain. All the samples were prepared by mixing polymer powders resulting from the utilization of $0.5 \mathrm{~mm}$ trapezoidal perforation sieve rings under the same controlled pressure of $16 \mathrm{MPa}$ at $200^{\circ} \mathrm{C}$ for $2 \mathrm{~h}$. 
At this point, it should be noted that it is valuable that new vitrimer blends with tunable thermomechanical properties can be generated by simply mixing the existing "waste" pure vitrimer powders without involving complex chemistry. Such simple method is eco-friendly and enables us to meet the requirement of different working conditions for practical engineering applications.

\subsection{Designability of the vitrimer blends}

Since the material combination used in the actual experiment is limited and the accuracy of the computational model has been validated, parametric studies could be conducted by using the model to further explore the designability of the vitrimer blends. In this sub-section, two design examples are studied where the rubbery modulus, glass transition temperature difference, and $\tan \delta$ breadth of the base vitrimers are designing parameters.

The first design example shown in Fig. 5 explores the possibility of decreasing the rubbery modulus of the vitrimer blends while enhancing their modulus at room temperature. Normally, we want a vitrimer possesses a high modulus at low temperature to meet the requirement of load-bearing capacity as a structural material. Also, a lower rubbery modulus is expected to meet the requirement of reprocessing under a low pressure. As shown in Fig. 5a and b, we create the base material 2 in the computational model to be the vitrimer to be modified, and the base material 1 is the modifier vitrimer with a lower rubbery modulus and higher glass transition temperature. The simulation results of the temperature-dependent storage modulus of the vitrimer blends are shown in Fig. $5 \mathrm{c}$, and the corresponding values at room temperature $\left(25{ }^{\circ} \mathrm{C}\right)$ and high temperature $\left(105{ }^{\circ} \mathrm{C}\right)$ are respectively recorded in Fig. $5 \mathrm{~d}$. It is
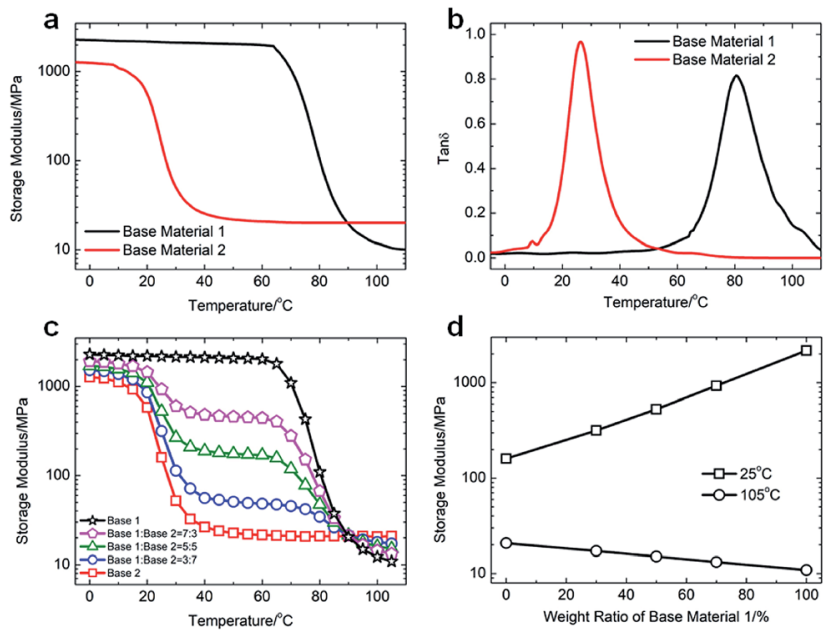

Fig. 5 Tunability of the modulus of the vitrimer blends. (a) Temperature dependence of the storage modulus of the designed base materials 1 and 2. (b) Temperature dependence of the $\tan \delta$ of the designed base materials 1 and 2. (c) Simulation results of the storage modulus of vitrimer blends with different compositions at elevated temperatures. (d) Variation of the storage modulus of vitrimer blends with composition of base material 1 at $25^{\circ} \mathrm{C}$ and $105^{\circ} \mathrm{C}$. seen that the room-temperature modulus of the blends increases linearly in the logarithm scale with the weight ratio of base material 1 , while the rubbery modulus at $105{ }^{\circ} \mathrm{C}$ linearly decreases, confirming the feasibility of tuning the material stiffness as designed.

The second example shows the possibility of tuning the miscibility of the vitrimer blends. For the completely miscible blends, only one glass transition can be observed in the DMA curves with a single $T_{\mathrm{g}}$ lying between the $T_{\mathrm{g}} \mathrm{s}$ of base components. ${ }^{36}$ As shown in Fig. $4 \mathrm{a}$ and b, there are two peaks in each $\tan \delta$ curve and one plateau in each storage modulus curve for the vitrimer blends created in the actual experiments, which indicates that the two base vitrimer components of the blends are not completely miscible. It is intriguing to explore if it is possible to modify the miscibility by decreasing the glass transition temperature difference and broadening the $\tan \delta$ curve breadth of the base vitrimer materials. As shown in Fig. 6a and b, two base vitrimers (base material 3 and 4) with close glass transition temperature and broad transition region are designed. The simulation results of the temperature-dependent storage modulus and $\tan \delta$ curves of the vitrimer blends are respectively shown in Fig. $6 \mathrm{c}$ and d. It is seen that upon mixing, there are no plateau in the storage modulus curve and only one peak in the $\tan \delta$ curve, which meets the goal of our design.

\subsection{Recyclability of the vitrimer blends}

Since the covalent bonds of vitrimer blends are only exchanged but not consumed during thermal processing, the resulting blends have the potential to be recycled for several times.

Fig. 7a compares the storage modulus curves for the $1^{\text {st }}$ and $3^{\text {rd }}$ generation of the recycled vitrimer blends obtained from the experiments. During each reprocessing cycle, the samples were
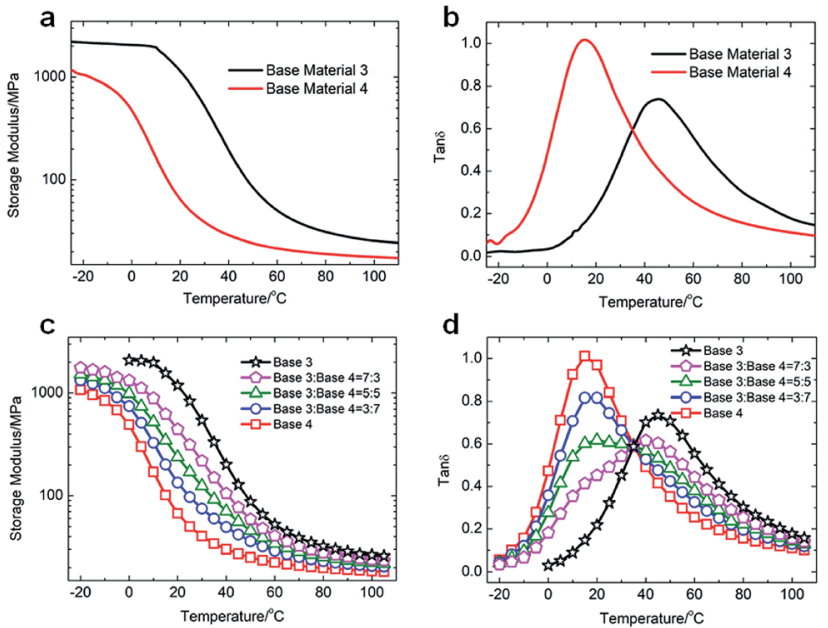

Fig. 6 Tunability of the miscibility of the vitrimer blends. (a) Temperature dependence of the storage modulus of the designed base materials 3 and 4. (b) Temperature dependence of the $\tan \delta$ of the designed base materials 3 and 4. (c) Simulation results of the storage modulus of vitrimer blends with different compositions at elevated temperatures. (d) Simulation results of the $\tan \delta$ of vitrimer blends with different compositions at elevated temperatures. 

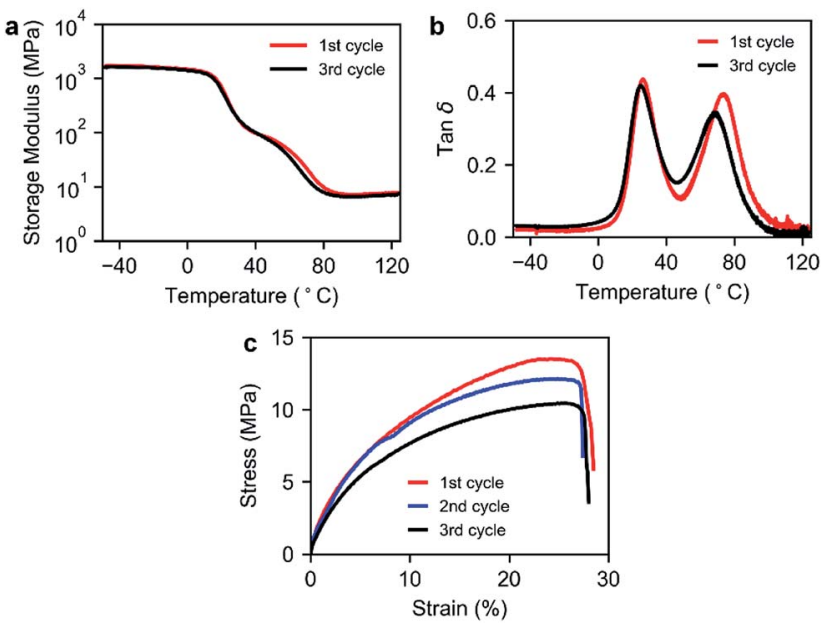

Fig. 7 Recyclability of the vitrimer blends. (a) Storage modulus vs. temperature of $1^{\text {st }}, 3^{\text {rd }}$ recycled samples. (b) Tan $\delta$ vs. temperature of $1^{\text {st }}, 3^{\text {rd }}$ recycled samples. (c) Uniaxial tensile test results of samples after being recycled for several generations.

thermally treated at $200{ }^{\circ} \mathrm{C}$ under a controlled pressure of $16 \mathrm{MPa}$ for $2 \mathrm{~h}$. The curves nearly overlapped with each other, except that the rubbery modulus was slightly decreased from 8.5 MPa to 7.3 MPa. The decrease of rubbery modulus might be resulted from the breakage of permanent covalent bonds during the vitrimer grinding. ${ }^{37}$ The glass transition temperatures show no apparent change after three reprocessing cycles (Fig. 7b). The stress-strain curves plotted in Fig. 7c illustrate that the mechanical properties of the recycled samples slightly degrade after each recycling treatment, yet compared to the $1^{\text {st }}$ recycled sample, the $3^{\text {rd }}$ generated sample maintains the comparable stretchability and $80 \%$ of the stiffness, showing the reasonably good recyclability.

\section{Conclusion}

In this work, we demonstrate a composite-based strategy to prepare vitrimer blends with tunable thermomechanical properties and reasonable recyclability by virtue of the dynamic covalent bond. Sufficient thermal processing with high processing temperature and long processing time may enhance the interface bonding, which accordingly induces higher modulus and greater stretchability. The tunable thermomechanical properties of vitrimer blends can be realized by adjusting the compositions of the base vitrimers, which offers a flexible and eco-friendly way to recycle the thermoset waste for practical engineering applications. To assist the blends design, a computational model accounting for the temperaturedependent thermomechanical behaviors of the pure vitrimers and the random distribution of the component vitrimer particles was developed to predict the thermomechanical properties of the recycled vitrimer blends. The validity of the computational model was proved by the good agreement between the experimental results and theoretical predictions of the thermomechanical properties of the recycled vitrimer blends with various compositions. The designability of the vitrimer blends was investigated by the parametric studies, showing the capability of increasing room-temperature modulus while decreasing the rubbery modulus, and generating vitrimers with good miscibility by decreasing glass transition temperature difference and broadening the tan $\delta$ curve breadth of pure base components. Finally, the reasonable recyclability of the vitrimer blends was verified. We expect that this composite-inspired blending method can be expanded to other transesterification-based vitrimers with excellent mechanical performance (high $T_{\mathrm{g}}$, stiffness and strength) to satisfy most requirements of practical applications.

\section{Conflicts of interest}

There are no conflicts to declare.

\section{Acknowledgements}

B. Z., M. L. D. and Q. G. gratefully acknowledge the support from the Agency for Science, Technology and Research (A*STAR) Public Sector Funding (PSF) (Project number 1521200086). Q. G. acknowledges the SUTD Start-up Research Grant. B. Z. acknowledges Northwestern Polytechnical University (NPU) Start-up Research Grant. Z. L. acknowledges RIE2020 AME Programmatic Grant (A18A1b0045) funded by A*STARSERC, Singapore. We also acknowledge support of the SUTD Digital Manufacturing and Design Center (DManD) for access to experimental and computational facilities.

\section{Notes and references}

1 Y. Huangfu, C. Liang, Y. Han, H. Qiu, P. Song, L. Wang, J. Kong and J. Gu, Compos. Sci. Technol., 2019, 169, 70-75.

2 H. Zhao, L. Chen, J. Yun, L. Tang, Z. Wen, X. Zhang and J. Gu, Eng. Sci., 2018, 2, 57-66.

3 J. Gu, C. Liang, J. Dang, W. Dong and Q. Zhang, RSC Adv., 2016, 6, 35809-35814.

4 Y. Tang, W. Dong, L. Tang, Y. Zhang, J. Kong and J. Gu, Compos. Commun., 2018, 8, 36-41.

5 L. Tang, J. Dang, M. He, J. Li, J. Kong, Y. Tang and J. Gu, Compos. Sci. Technol., 2019, 169, 120-126.

6 S.-C. Lin and E. M. Pearce, High performance thermosets: chemistry, properties, applications, Hanser Munich, 1994.

7 J. G. Drobny, Polymers for electricity and electronics: materials, properties, and applications, John Wiley \& Sons, 2012.

8 A. Fangareggi and L. Bertucelli, in Thermosets, Elsevier, 2012, pp. 254-288.

9 S. J. Pickering, Composites, Part A, 2006, 37, 1206-1215.

10 P. Pissis, Thermoset nanocomposites for engineering applications, iSmithers Rapra Publishing, 2007.

11 D. Montarnal, M. Capelot, F. Tournilhac and L. Leibler, Science, 2011, 334, 965-968.

12 M. Capelot, D. Montarnal, F. Tournilhac and L. Leibler, J. Am. Chem. Soc., 2012, 134, 7664-7667.

13 W. Denissen, J. M. Winne and F. E. Du Prez, Chem. Sci., 2016, 7, 30-38. 
14 K. Yu, Q. Shi, M. L. Dunn, T. J. Wang and H. J. Qi, Adv. Funct. Mater., 2016, 26, 6098-6106.

15 K. Yu, P. Taynton, W. Zhang, M. L. Dunn and H. J. Qi, RSC Adv., 2014, 4, 10108-10117.

16 C. Luo, X. Shi, Z. Lei, C. Zhu, W. Zhang and K. Yu, Polymer, 2018, 153, 43-51.

17 X. Yang, Y. Guo, X. Luo, N. Zheng, T. Ma, J. Tan, C. Li, Q. Zhang and J. Gu, Compos. Sci. Technol., 2018, 164, 59-64.

18 W. Denissen, G. Rivero, R. Nicolaÿ, L. Leibler, J. M. Winne and F. E. Du Prez, Adv. Funct. Mater., 2015, 25, 2451-2457.

19 Y.-X. Lu and Z. Guan, J. Am. Chem. Soc., 2012, 134, 1422614231.

20 Y.-X. Lu, F. Tournilhac, L. Leibler and Z. Guan, J. Am. Chem. Soc., 2012, 134, 8424-8427.

21 J. J. Cash, T. Kubo, A. P. Bapat and B. S. Sumerlin, Macromolecules, 2015, 48, 2098-2106.

22 O. R. Cromwell, J. Chung and Z. Guan, J. Am. Chem. Soc., 2015, 137, 6492-6495.

23 J. C. Lai, J. F. Mei, X. Y. Jia, C. H. Li, X. Z. You and Z. Bao, Adv. Mater., 2016, 28, 8277-8282.

24 M. M. Obadia, B. P. Mudraboyina, A. Serghei, D. Montarnal and E. Drockenmuller, J. Am. Chem. Soc., 2015, 137, 60786083.

25 A. Chao, I. Negulescu and D. Zhang, Macromolecules, 2016, 49, 6277-6284.
26 P. Taynton, H. Ni, C. Zhu, K. Yu, S. Loob, Y. Jin, H. J. Qi and W. Zhang, Adv. Mater., 2016, 28, 2904-2909.

27 P. Taynton, K. Yu, R. K. Shoemaker, Y. Jin, H. J. Qi and W. Zhang, Adv. Mater., 2014, 26, 3938-3942.

28 N. Zheng, Z. Fang, W. Zou, Q. Zhao and T. Xie, Angew. Chem., Int. Ed., 2016, 55, 11421-11425.

29 Z. P. Zhang, M. Z. Rong and M. Q. Zhang, Adv. Funct. Mater., 2018, 28, 1706050.

30 K. Yu, P. Taynton, W. Zhang, M. L. Dunn and H. J. Qi, RSC $A d v .$, 2014, 4, 48682-48690.

31 Z. Pei, Y. Yang, Q. Chen, E. M. Terentjev, Y. Wei and Y. Ji, Nat. Mater., 2014, 13, 36-41.

32 B. Zhang, K. Kowsari, A. Serjouei, M. L. Dunn and Q. Ge, Nat. Commun., 2018, 9, 1831.

33 K. Yu, Q. Ge and H. J. Qi, Nat. Commun., 2014, 5, 3066.

34 C. Yuan, Z. Ding, T. Wang, M. L. Dunn and H. J. Qi, Smart Mater. Struct., 2017, 26, 105027.

35 K. Yu, Q. Shi, H. Li, J. Jabour, H. Yang, M. L. Dunn, T. Wang and H. J. Qi, J. Mech. Phys. Solids, 2016, 94, 1-17.

36 N. Avramova, in Plastics Additives, Springer, 1998, pp. 513518.

37 E. B. Stukalin, L.-H. Cai, N. A. Kumar, L. Leibler and M. Rubinstein, Macromolecules, 2013, 46, 7525-7541. 